\title{
Translation and Validation of Malay Version of the Simplified Diabetes Knowledge Test
}

Mohd Aznan Bin Md Aris ${ }^{1}$, Umair Bin Othman ${ }^{2}$, Nur-Hidayah Bt Abdul Rahim², Hafizah Bt Pasi ${ }^{3}$

${ }^{1}$ Department of Family Medicine, International Islamic University Malayisa

${ }^{2}$ Kulliyyah of Medicine, International Islamic University Malaysia

${ }^{3}$ Department of Community Medicine, Kulliyyah of Medicine, International Islamic University Malaysia

Presenter: Mohd Aznan Md Aris

Introduction: Type 2 diabetes mellitus is a non-communicating disease with an increasing trend in Malaysia. One of the most important aspects in the selfmanagement of diabetes mellitus is the assessment of knowledge among diabetes patients. This study aimed to translate and validate the Malay version of Modified Diabetes Knowledge Test (DKT) questionnaire. Methods: A standard back-to-back translation procedure was used to translate the simplified version of the DKT questionnaire from the original version. A total of 120 outpatients with type 2 diabetes mellitus from the government primary care clinic were selected to answer the questionnaire which consists of socio-demographic and diabetes characteristics questions as well as the 20-item DKT questions. Reliability and validity were tested with Cronbach's alpha and Exploratory Factor Analysis. Results: The Cronbach's alpha for the translated questionnaire was acceptable $(a=0.573)$ with the Cronbach's alpha excluding each item ranged from 0.517 to 0.602 . Factor analysis with Varimax rotation identified 6 domains for the 18 items, with 2 to 6 items in each domain. Conclusion: This version of simplified DKT is proven to be valid and reliable, and can be used with caution as a brief assessment of knowledge among diabetic patients specifically tailored for Malaysian population. 